\title{
Behavior Therapy Augments Response of Patients With Obsessive-Compulsive Disorder Responding to Drug Treatment
}

\author{
Nienke H. Tenneij, Ph.D.; Harold J. G. M. van Megen, M.D.; \\ Damiaan A. J. P. Denys, M.D.; and Herman G. M. Westenberg, Ph.D.
}

Objective: In many patients with obsessivecompulsive disorder (OCD), residual symptoms persist despite a clinically meaningful response. The objective of this study was to examine whether addition of behavior therapy would augment treatment outcome in these patients.

Method: Ninety-six patients with DSM-IV OCD who had responded to 3 months of drug treatment were randomly assigned to either receive addition of behavior therapy or continue on drug treatment alone for 6 months. Patients who continued on drug treatment alone eventually received addition of behavior therapy for 6 months. Data were gathered from October 1998 to June 2002.

Results: OCD patients who received addition of behavior therapy showed a greater improvement in obsessive-compulsive symptoms (YaleBrown Obsessive Compulsive Scale [Y-BOCS] score change $=-3.9$ in the completers sample) than those who continued on drug treatment alone (Y-BOCS score change $=+3.9$ for completers) . Significantly more patients who received addition of behavior therapy were in remission compared with those who continued on drug treatment alone ( $\mathrm{p}<.0001$ for completers). Patients who received behavior therapy after 6 months of drug treatment alone showed a nonsignificant decline in obsessive-compulsive symptoms (Y-BOCS score change $=-2.7$ for completers); however, the remission rate found in this group was comparable to the remission rate found in the group of patients receiving addition of behavior therapy directly after responding to drug treatment.

Conclusion: The results indicate that addition of behavior therapy is beneficial for patients who have responded to drug treatment. The data also suggest that the effect is greater when behavior therapy is added immediately after attainment of the drug response.

(J Clin Psychiatry 2005;66:1169-1175)

Received June 21, 2004; accepted March 7, 2005. From the Department of Psychiatry, Rudolf Magnus Institute of Neuroscience, University Medical Center Utrecht, Utrecht, the Netherlands. This work was supported, in part, by unrestricted grants from GlaxoSmithKline, Zeist, the Netherlands, and Wyeth Pharmaceuticals, Hoofddorp, the Netherlands.

The authors report no other financial relationship or affiliation relevant to the subject of this article.

Corresponding author and reprints: Nienke H. Tenneij, Ph.D., Vrije Universiteit Amsterdam, Developmental Psychology, Van der Boechorststraat, 11081 BT Amsterdam, the Netherlands (e-mail:nh.tenneij@psy.vu.nl).

$\mathbf{O}$ bsessive-compulsive disorder (OCD) is a chronic and disabling condition in which obsessions and compulsions disrupt normal daily functioning. Community surveys have estimated the lifetime prevalence of OCD to be between $2 \%$ and $3 \%$. ${ }^{1,2}$ The mainstays of treatment for OCD are drug treatment, notably with serotonin reuptake inhibitors (SRIs), and behavior therapy (also referred to as cognitive-behavioral therapy). Most guidelines propose behavior therapy for mild-to-moderate cases of OCD and drug treatment for moderate-to-severe cases of OCD as first-line treatment. ${ }^{3}$ A combination of drug and behavior therapy has been suggested as optimal treatment for all OCD patients. ${ }^{4}$ Surprisingly few studies, however, have been conducted to validate this recommendation. To our knowledge, only 7 controlled studies have investigated the efficacy of combination therapy.

Marks et al. ${ }^{5}$ compared the efficacy of clomipramine or placebo alone for 4 to 7 weeks with the subsequent addition of behavior therapy or relaxation treatment for 3 months. In that study, as well as in their subsequent study, ${ }^{6}$ the combination of clomipramine and behavior therapy tended to be superior to either treatment alone. The adjuvant role of clomipramine to behavior therapy, however, was small and transient. Similar results were also reported by Cottraux et al., ${ }^{7}$ who found a short-lived superiority for the combination therapy compared with fluvoxamine or behavior therapy alone. Foa et al., ${ }^{8}$ using a randomized, placebo-controlled design, found intensive behavior therapy and the combination of behavior therapy and clomipramine superior to clomipramine alone, while all active treatments were superior to placebo. The addition of clomipramine to behavior therapy, however, had no additive effect in this study. Hohagen et al. ${ }^{9}$ found that the combi- 
nation of fluvoxamine and behavior therapy was superior to behavior therapy alone, but van Balkom et al. ${ }^{10}$ failed to find a superior effect for this combination over behavior therapy. In the latter study, however, a sequential design was used in which behavior therapy was added to fluvoxamine after 8 weeks of drug treatment. Finally, Kampman et al. ${ }^{11}$ studied the effect of combination therapy in OCD patients who had not responded to drug treatment. They found that addition of behavior therapy in patients who had not responded to a 12-week fluoxetine treatment was effective. Unfortunately, a control group continuing on fluoxetine treatment alone was lacking in this study.

Generally speaking, the data reported so far are not unequivocal and do not permit firm conclusions as to the superior efficacy of combination therapy in a random OCD sample. Differences in design, treatment procedure, and patient selection criteria (OCD subtype and severity) may account in part for the reported discrepancies.

Although combination therapy has been advocated as optimal treatment for OCD patients, including those who have already responded to drug treatment, it is surprising that no controlled study has been conducted to validate this recommendation. The aim of the present study was, therefore, to study the efficacy of the combination of behavior therapy and drug treatment in OCD patients responding to SRIs. This is a relevant issue because behavior therapy is expensive and time-consuming and not always readily available. A related question was therefore whether the timing of the addition of behavior therapy, namely, 3 or 9 months after the start of drug treatment, would have an effect on treatment outcome.

\section{METHOD}

\section{Design}

OCD patients who showed a reduction of at least $25 \%$ on the Yale-Brown Obsessive Compulsive Scale (Y-BOCS $)^{12,13}$ after 3 months of drug treatment were randomly allocated to either continue drug treatment (drug treatment group: DT) or receive behavior therapy in addition to their drug treatment (combination therapy group: CT) for 6 months. Patients allocated to the DT group subsequently received addition of behavior therapy for another period of 6 months (delayed combination therapy group: dCT).

\section{Subjects}

Ninety-six adult outpatients, with a mean \pm SD age of $36 \pm 11.1$ years, who had a primary diagnosis of OCD according to DSM-IV ${ }^{14}$ entered the study. Data were gathered from October 1998 to June 2002. Patients were recruited from a sample of 150 OCD patients who participated in a double-blind trial comparing the efficacy of paroxetine and venlafaxine. ${ }^{15}$ All patients gave written informed consent for participation in this study, which was

\begin{tabular}{|c|c|c|c|}
\hline Characteristic & $\mathrm{CT}(\mathrm{N}=47)$ & DT $(\mathrm{N}=49)$ & $\mathrm{p}$ Value \\
\hline Female, $\%$ & 62 & 67 & .56 \\
\hline In $(\mathrm{SD}), \mathrm{y}$ & $36.7(12)$ & $36.7(10)$ & .99 \\
\hline t, mean (SD), y & $0.3(7)$ & $0.4(10)$ & .93 \\
\hline mean (SD), y & & & .82 \\
\hline & 1 & & .93 \\
\hline $\mathrm{H}$ & 6 & & .22 \\
\hline HAM-D score, mean (SD) & $5.4(5)$ & $3.9(3)$ & .08 \\
\hline aroxetine treatment, $\%$ & 45 & 53 & .41 \\
\hline $\mathrm{y}, \%$ & 7 & & .38 \\
\hline & & & .55 \\
\hline Previous behavior therapy, $\%$ & 32 & 39 & .44 \\
\hline
\end{tabular}

approved by the University of Utrecht Medical Ethical Review Committee. Patients participating in this trial were treated for 12 weeks at fixed doses of $300 \mathrm{mg} /$ day of venlafaxine or $60 \mathrm{mg} / \mathrm{day}$ of paroxetine. Patients with comorbid Axis I disorders (major depressive disorder, bipolar disorder, schizophrenia or any other psychotic disorder, tic disorder, or substance-related disorder during the past 6 months), a Hamilton Rating Scale for Depression (HAM-D $)^{16}$ score higher than 16, or cognitive and/or behavior therapy within 6 months prior to randomization were excluded from this trial. One hundred nine of the 150 patients had a reduction of at least $25 \%$ in Y-BOCS score at endpoint. The mean \pm SD Y-BOCS total score in this sample of 109 patients declined from $25.4 \pm 5.3$ before treatment to $14.3 \pm 5.8$ at endpoint. The mean percentage symptom reduction on drug treatment in this sample was $44 \%$.

The demographic characteristics of the sample are presented in Table 1. No significant differences were observed across the 2 conditions at baseline. Eighty patients completed the 6-month treatment period. Thirteen patients from the CT group and 3 from the DT group dropped out. There was no difference in OCD symptom severity, depressive symptoms, anxiety symptoms, illness duration, or age between dropouts and completers. Forty-six patients from the DT group entered the dCT phase, of which 25 patients completed this phase. Fifteen patients dropped out, and no endpoint data were available for analysis for 6 patients.

\section{Treatment}

Patients in the DT condition had 4 appointments of 30 minutes with a psychiatrist. During these appointments, psychiatric and somatic evaluations took place, but no formal cognitive interventions or exposure with response prevention instructions were supplied. Appointments were recorded on tape and checked by an independent psychiatrist for adherence to the instructions. Overall, 
adherence to the instructions was good to excellent. Patients in the CT condition received 18 sessions of behavior therapy by an experienced therapist. Each session lasted 45 minutes. Standardization of the behavior therapy was ensured by using a detailed treatment manual and regular supervision sessions. The format of exposure in vivo and response prevention was gradual self-controlled exposure in vivo with gradual self-imposed response prevention. The first therapy session was used to explain the procedure and to make an inventory of the symptoms. Patients were asked to record their symptoms daily. In the next session, symptoms were hierarchically ordered and the first exposure and response prevention assignments were discussed. In subsequent sessions, the results of exposure and response prevention assignments were discussed and new exposure exercises were planned. In the last 2 sessions, attention was focused on relapse prevention, taking into account anticipated difficulties and the possible strategies to cope with these foreseeable situations.

Completers in the combination therapy groups, with the exception of 4 patients, received 18 sessions of behavior therapy. The 4 patients were in remission after 7 , 10,12 , and 15 sessions, respectively, rendering behavior therapy no longer useful.

All sessions were recorded on tape, and a random selection of these tapes was checked for adherence to the treatment manual. Sessions could be judged from 0 (no adherence at all) to 5 (excellent adherence to the manual). Overall, the adherence to the manual was good (mean \pm $\mathrm{SD}=4.37 \pm 0.78$ ).

\section{Measures}

The Y-BOCS was used to assess severity of obsessivecompulsive symptoms. The 17-item HAM-D was used to measure the severity of depressive symptoms, and the Hamilton Rating Scale for Anxiety (HAM-A) ${ }^{17}$ was used to measure anxiety symptoms. The Y-BOCS and HAM-A were administered at baseline, week 9, week 18, and week 27, and the HAM-D was administered at baseline and week 27. Trained investigators, blind to the patients' assignment condition, carried out the measurements. Patients in the dCT condition were also assessed at week 54. Assessments in the first and second phases were performed by different investigators.

\section{Statistical Analyses}

Pretreatment differences in demographic and clinical variables between the 2 conditions (DT and CT) were analyzed with Student $t$ tests or $\chi^{2}$ tests.

In the completers sample, treatment effects for OCD and anxiety symptoms were analyzed with multivariate analysis of variance (ANOVA) with time (baseline through week 27) as within factor and drug (paroxetine or venlafaxine) and treatment (CT or DT) as between factors. In case of significant multivariate effects, univariate analyses were subsequently performed for the separate outcome measures. Furthermore, significant time effects within groups were analyzed using paired $t$ tests, and interaction effects were analyzed using $t$ tests for independent groups.

Intent-to-treat (ITT) analyses with last-observationcarried-forward analyses were conducted for all patients with at least 1 assessment after baseline. Treatment effects for the Y-BOCS total score and HAM-A were analyzed with time (baseline and week 27) and treatment (CT or DT) as between factors.

To examine the effect of duration of drug treatment prior to behavioral therapy on outcome, specifically, 3 or 9 months after commencement of drug treatment, the results of the addition of behavioral therapy in the CT and $\mathrm{dCT}$ groups were compared using multivariate ANOVA with time (baseline vs. week 27 in the CT group and week 54 in the $\mathrm{dCT}$ group) as within factor, and treatment as between factor.

To investigate the clinical significance of combination therapy as opposed to drug treatment alone, and to compare the efficacy of combination therapy 3 or 9 months after the onset of drug treatment, we calculated the remission rates and compared the percentage of patients in remission in the different conditions using the $\chi^{2}$ test. Remission was defined as a total Y-BOCS score of 8 or less. ${ }^{18}$

\section{RESULTS}

\section{Combination Therapy Versus Drug Therapy}

When the primary outcome measures (Y-BOCS and HAM-A) of the completers sample were entered into the analysis, a statistically significant main effect for time $(\mathrm{F}=$ 13.08, $\mathrm{df}=3,648 ; \mathrm{p}<.001)$ and treatment $(\mathrm{F}=5.05, \mathrm{df}=$ $1,72 ; \mathrm{p}<.03)$, but not for drug $(\mathrm{F}=0.594$, $\mathrm{df}=1,72$; $\mathrm{p}=.44)$, was observed. In addition, a significant time-bytreatment interaction $(\mathrm{F}=10.67, \mathrm{df}=3,648 ; \mathrm{p}<.001)$, but no significant time-by-drug $(\mathrm{F}=1.32, \mathrm{df}=3,648 ; \mathrm{p}=.27)$ or drug-by-treatment $(\mathrm{F}=.716, \mathrm{df}=1,72 ; \mathrm{p}=.40)$ interaction, was found. Therefore, the data for paroxetine and venlafaxine were combined for subsequent analyses.

The subsequent multivariate effects were analyzed with univariate repeated-measures ANOVA. The results are depicted in Table 2 . Analyses by outcome measure revealed a statistically significant main effect for time on the Y-BOCS total score $(\mathrm{F}=6.59, \mathrm{df}=3,225 ; \mathrm{p}<.001)$, the Y-BOCS obsessions $(\mathrm{F}=3.15, \mathrm{df}=3,225 ; \mathrm{p}=.03)$ and compulsions $(\mathrm{F}=6.45, \mathrm{df}=3,225 ; \mathrm{p}<.001)$ subscales, and the HAM-A $(\mathrm{F}=21.79, \mathrm{df}=3,225 ; \mathrm{p}<.001)$. A significant time-by-treatment interaction for the Y-BOCS total score $(\mathrm{F}=11.54, \mathrm{df}=3,225 ; \mathrm{p}<.001)$ and the Y-BOCS obsessions $(\mathrm{F}=7.24, \mathrm{df}=3,225 ; \mathrm{p}<.001)$ and compulsions $(\mathrm{F}=9.86, \mathrm{df}=3,225 ; \mathrm{p}<.001)$ subscales, but not for the HAM-A $(\mathrm{F}=.837, \mathrm{df}=3,225 ; \mathrm{p}=.48)$, was found. Time effects within treatment conditions were analyzed 


\begin{tabular}{|c|c|c|c|c|c|}
\hline \multirow{3}{*}{ Measure } & \multirow{2}{*}{\multicolumn{4}{|c|}{ Week }} & \multirow[b]{3}{*}{ Endpoint } \\
\hline & & & & & \\
\hline & 0 & 9 & 18 & 27 & \\
\hline \multicolumn{6}{|c|}{ Y-BOCS } \\
\hline \multicolumn{6}{|c|}{ Obsessions } \\
\hline $\mathrm{CT}$ & $7.13(2.54)$ & $7.87(3.20)$ & $6.91(3.92)$ & $5.34(4.40)^{*}$ & $5.52(4.19)^{*}$ \\
\hline DT & $7.48(3.55)$ & $8.82(4.27) *$ & $8.82(3.96)^{*}$ & $9.30(4.15)$ & $8.91(4.19)^{*}$ \\
\hline \multicolumn{6}{|c|}{ Compulsions } \\
\hline $\mathrm{CT}$ & $7.34(2.91)$ & $7.84(3.69)$ & $6.66(3.50)$ & $5.22(3.82)^{*}$ & $6.23(4.13)^{*}$ \\
\hline DT & $7.02(3.86)$ & $9.41(4.84)$ & $9.27(4.39)$ & $9.09(4.98)$ & $8.66(5.03)^{*}$ \\
\hline \multicolumn{6}{|c|}{ Total score } \\
\hline CT & $14.47(4.93)$ & $15.72(5.64)$ & $13.56(6.62)$ & $10.56(7.77)^{*}$ & $11.76(7.66)^{*}$ \\
\hline DT & $14.50(6.17)$ & $18.23(7.99)^{*}$ & $18.09(7.76)^{*}$ & $18.36(7.95)^{*}$ & $17.56(8.13)^{*}$ \\
\hline \multicolumn{6}{|l|}{ HAM-A } \\
\hline CT & $5.44(3.50)$ & $10.75(5.89)^{*}$ & $9.38(6.64)^{*}$ & $9.66(7.95)^{*}$ & $9.16(7.62)^{*}$ \\
\hline DT & $5.93(3.37)$ & $10.07(6.69)^{*}$ & $9.16(6.99)^{*}$ & $10.89(7.85)^{*}$ & $10.83(7.80)^{*}$ \\
\hline \multicolumn{6}{|l|}{ HAM-D } \\
\hline CT & $4.88(3.89)$ & & & $5.47(5.32)$ & $5.62(5.45)$ \\
\hline DT & $3.96(2.99)$ & & & $6.47(5.78)$ & $6.35(5.67)$ \\
\hline \multicolumn{6}{|c|}{$\begin{array}{l}\text { aData are presented as mean }(\mathrm{SD}) \text {. } \\
\text { *Significantly }(\mathrm{p} \leq .001) \text { different from baseline (week 0) with 2-tailed t tests. } \\
\text { Abbreviations: HAM-A = Hamilton Rating Scale for Anxiety, HAM-D = Hamilton Rating Scale for } \\
\text { Depression, Y-BOCS = Yale-Brown Obsessive Compulsive Scale. }\end{array}$} \\
\hline
\end{tabular}

using paired t tests (baseline vs. weeks 9, 18, and 27). Analyses revealed a significant increase in Y-BOCS score in the DT group (+3.9) and a significant decrease (-3.9) in the CT group. In both groups, a significant increase in anxiety symptoms was noted.

The change in Y-BOCS total score for both groups is depicted in Figure 1. Post hoc analysis using $t$ tests for independent groups revealed a statistically significant difference between the treatment conditions at week 18 $(\mathrm{t}=2.87, \mathrm{p}<.01)$ and week $27(\mathrm{t}=4.59, \mathrm{p}<.001)$.

The univariate repeated-measures analysis for the HAM-D showed a main effect for time $(\mathrm{F}=9.032$, df $=$ $1,75 ; \mathrm{p}=.004)$ but no time-by-treatment condition interaction $(\mathrm{F}=2.94$, df $=1,75 ; \mathrm{p}=.091)$. HAM-D scores increased slightly during treatment, but the effect was not different between the treatment conditions.

Almost identical results were obtained for the ITT sample. For the primary outcome measures (Y-BOCS and HAM-A), a multivariate main effect for time $(\mathrm{F}=21.53$, $\mathrm{df}=1,178 ; \mathrm{p}<.001)$, but not for drug $(\mathrm{F}=0.387$, df $=$ $1,89 ; \mathrm{p}=.54)$ or treatment $(\mathrm{F}=1.41, \mathrm{df}=1,89 ; \mathrm{p}=.24)$, was found. In addition, a significant treatment-by-time interaction $(\mathrm{F}=16.99$, $\mathrm{df}=1,178 ; \mathrm{p}<.001)$, but no timeby-drug $(\mathrm{F}=0.294$, df $=1,178 ; \mathrm{p}=.59)$ or treatmentby-drug $(\mathrm{F}=1.19$, df $=1,89 ; \mathrm{p}=.28)$ interaction, was observed.

\section{Combination Therapy Versus \\ Delayed Combination Therapy}

The repeated-measures ANOVA for completers with time (before vs. after behavior therapy) as within factor and treatment (CT vs. dCT) as between factor revealed a statistically significant main effect for time $(F=12.39$,
Figure 1. Mean Y-BOCS Scores at Weeks 0, 9, 18, and 27 for Drug Treatment and Combination Therapy

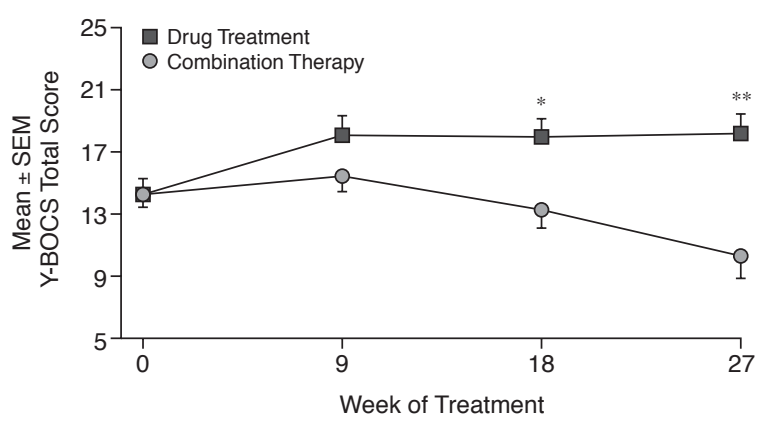

$* \mathrm{p}<.01$ for drug treatment vs. combination therapy.

$* * \mathrm{p}<.001$ for drug treatment vs. combination therapy.

Abbreviation: Y-BOCS = Yale-Brown Obsessive Compulsive Scale

$\mathrm{df}=1,57 ; \mathrm{p}<.01)$, but no interaction between time and treatment $(\mathrm{F}=0.337, \mathrm{df}=1,57 ; \mathrm{p}=.56)$. The time effects within treatment conditions were analyzed by using paired tests. The results are depicted in Table 3. The symptoms in the dCT group decreased $(-2.7$ on the Y-BOCS in completers), but this effect just failed to reach statistical significance.

Similar results were obtained for the ITT sample. A main effect for time $(F=4.30$, df $=1,81 ; \mathrm{p}<.05)$, but no interaction between time and treatment $(\mathrm{F}=0.98$, df $=1,81 ; p=.33)$, was observed.

\section{Remission Rates}

Percentages of patients in remission in the completers and ITT samples, respectively, were as follows: CT group, $53 \%(18 / 34)$ and 40\% (19/47); dCT group, 40\% (10/25) 
Table 3. Y-BOCS Ratings at Baseline and After Treatment for the Combination Therapy (CT) and Delayed Combination Therapy (dCT) Groups for the Completers and Intent-to-Treat Samples ${ }^{\mathrm{a}}$

\begin{tabular}{lccccc}
\hline & \multicolumn{2}{c}{ Completers $(\mathrm{N}=59)$} & & \multicolumn{2}{c}{$\begin{array}{c}\text { Intent-to-Treat } \\
\text { Sample }\end{array}$} \\
\cline { 2 - 3 } \cline { 5 - 6 } Group & Baseline & Posttreatment & & Baseline & Posttreatment \\
\hline CT & $13.88(5.34)$ & $10.09(7.77)^{*}$ & & $14.26(5.08)$ & $11.74(7.62)^{* * *}$ \\
dCT & $14.52(4.66)$ & $11.80(7.41)$ & & $15.06(5.86)$ & $14.17(8.25)$ \\
\hline
\end{tabular}

${ }^{\mathrm{a} D a t a}$ are presented as mean (SD).

Intent-to-treat $\mathrm{N}$ includes 47 patients in the $\mathrm{CT}$ group and 36 patients in the dCT group (46 patients from the drug treatment group entered the dCT phase; for 6 , no endpoint data were available, and for 4 , only a baseline assessment was available).

*Significantly different $(\mathrm{p}=.005)$ from baseline with paired t test.

**Significantly different $(\mathrm{p}=.001)$ from baseline with paired $\mathrm{t}$ test.

Abbreviations: Y-BOCS = Yale-Brown Obsessive Compulsive Scale.

and $36 \%(13 / 36)$; DT group, $11 \%(5 / 45)$ and $15 \%(7 / 48)$ (data from the DT phase were unavailable for 1 patient). There was a statistically significant difference between the CT and DT groups with regard to percentages of patients in remission for both the completers $(\mathrm{df}=2$, $\mathrm{p}<.0001)$ and ITT samples $(\mathrm{df}=2, \mathrm{p}=.008)$. Significantly more patients in the CT group were in remission compared with the DT group. When the timing of addition was examined, no statistically significant differences in the percentages of patients in remission between subjects in the CT and $\mathrm{dCT}$ conditions were found in either the completers $(\mathrm{df}=2, \mathrm{p}=.19)$ or the ITT sample $(\mathrm{df}=2$, $\mathrm{p}=.34)$.

\section{Dropout Rates}

Twenty-eight patients prematurely discontinued the combination therapy. The most frequently reported reason for discontinuation in this group was lack of motivation to continue behavior therapy $(\mathrm{N}=20)$. Most patients attributed their lack of motivation to the substantial improvement already attained during prior drug treatment. They were no longer willing to invest in weekly sessions of behavior therapy and the assigned homework. Three other patients dropped out because they stopped taking their medication. Five patients were no longer eligible because they needed treatments for other medical or psychological problems. Three patients prematurely discontinued the drug treatment condition, 2 dropped out due to protocol violations, and 1 discontinued for unknown reasons.

\section{DISCUSSION}

The present study was designed to answer the clinically important question concerning the benefit of addition of behavior therapy in OCD patients responding to drug treatment. The study reveals that augmentation with behavior therapy is valuable. Patients receiving combination therapy after responding to drug treatment showed a significantly greater improvement in Y-BOCS score than those who continued on drug treatment alone. The mean difference in score between the 2 conditions at endpoint after 6 months was 7.8 in the completers sample and 5.8 in the ITT sample. The beneficial effect of combination therapy is particularly demonstrated by the percentage of patients in remission; $11 \%$ of the patients in the drug treatment group versus $53 \%$ in the combination therapy group could be considered in remission at endpoint. This is a clinically important finding, in view of the notion that the majority of OCD patients responding to drug treatment have residual symptoms, whereas only a minority are in remission. ${ }^{19}$

The data also show that addition of behavior therapy is affected by the timing of the addition with respect to the duration of drug treatment. When behavior therapy was added 3 months after the onset of drug treatment, a greater effect was obtained than when the addition was commenced after 9 months of drug treatment. However, when the percentage of patients in remission was used as the outcome measure, the timing of the addition had no effect on outcome; both combination therapy groups were superior to drug treatment alone. Thus, addition of behavior therapy may be beneficial irrespective of the timing, but the optimal effect seems to be attained immediately after response to drug treatment, which is typically after 12 weeks.

To the best of our knowledge, this is the first study providing evidence for the prevailing treatment guidelines for $\mathrm{OCD},{ }^{3}$ which recommend SRIs as initial treatment for patients with moderate-to-severe OCD and addition of behavior therapy for those cases that are responding to medication. Thus far, only 7 controlled studies ${ }^{5-11}$ in adult OCD patients have compared the effect of combination therapy with either behavior therapy or drug treatment alone. In 4 studies, ${ }^{6-9}$ patients were randomly assigned to combination therapy, drug treatment, and/or behavior therapy alone. In 3 of these studies, ${ }^{6-8}$ combination therapy was not superior to behavior therapy.

Marks et al. ${ }^{6}$ found that clomipramine compared with placebo as an adjuvant to behavior therapy yielded a limited and transient benefit on some outcome measures. Behavior therapy was the most potent treatment condition in the study. A limitation of the latter study is that patients who previously had not responded to behavior therapy were excluded from the study, which might explain the superiority of behavior therapy. Similar results were reported by Cottraux et al. ${ }^{7}$ using fluvoxamine as drug treatment. Recently, Foa et al. ${ }^{8}$ found that combination therapy was superior to clomipramine, but not different from behavior therapy. A limitation of the study was the large number of subjects who dropped out after learning their assignment. In contrast, Hohagen et al. ${ }^{9}$ found that the combination of fluvoxamine and behavior therapy was superior to behavior therapy and placebo, particularly when compulsions dominated the clinical picture and 
when patients had secondary depression. Unfortunately, a drug-alone condition was lacking in this study, and the duration of treatment was relatively short (8 weeks). These findings were recently confirmed in a study in children and adolescents with OCD. ${ }^{20}$ In that study, sertraline plus behavior therapy was superior to either treatment alone.

In 2 other studies, ${ }^{5,10}$ drug treatment and combination therapy were given sequentially, but no distinction was made between responders and nonresponders to drug treatment. Van Balkom et al. ${ }^{10}$ added behavior therapy or cognitive therapy after 8 weeks of fluvoxamine treatment and compared the results to behavior therapy or cognitive therapy alone. They found no differences between the treatment conditions. The study did not include a drugalone group to control for delayed drug effects of fluvoxamine. Marks et al. ${ }^{5}$ randomly assigned OCD patients to clomipramine or placebo and added behavior therapy or relaxation treatment after 4 to 7 weeks of drug treatment. Clomipramine-treated patients improved significantly more than did placebo-treated patients, but this effect was present only in patients with high depression scores at the outset. Addition of behavior therapy had a slight, nonsignificant additive effect.

Finally, Kampman et al. ${ }^{11}$ studied the efficacy of combination therapy in nonresponders to fluoxetine treatment and showed that the addition of behavior therapy in patients not responding to fluoxetine resulted in a statistically significant reduction in OCD symptoms. A limitation of this study was the lack of a drug control group. Although the drug treatment period was sufficiently long, it cannot be ruled out that initial nonresponders to fluoxetine improved subsequently due to continued drug treatment.

Two differences between the design of the present study and the other combination studies are noteworthy. Most importantly, all patients in the present study had responded to drug treatment before behavior therapy was added, which comes close to clinical practice, where treatment of patients with moderate-to-severe OCD is usually commenced with drug treatment. Furthermore, patients in this study were treated for a sufficiently long period with an effective drug before behavior therapy was added.

Several study limitations merit consideration. First, the dropout rate in the combination treatment groups (30\%) was substantially higher than in the drug treatment condition (6\%), but not higher than those seen in most other studies using behavior therapy. ${ }^{4,8}$ The main reason for premature discontinuation in the combination treatment group was lack of motivation. Most of these patients had gained substantial improvement or were in remission at the time of discontinuation and were no longer willing to commit themselves to time-consuming sessions and homework assignments. Second, patients with comorbid depression were excluded from this study. Although this exclusion is the rule rather than the exception in drug trials in order to avoid an effect of drugs on OCD symptoms by their effect on mood, ${ }^{5}$ in clinical practice the majority of OCD patients present with depressive symptoms secondary to OCD and one third of the patients qualify for current major depressive disorder. ${ }^{21,22}$ By the same token, SRIs are first-line treatment in moderate-tosevere cases of OCD. Third, this study focused on patients (partially) responding to drug treatment and was not designed to answer the question of whether addition of behavior therapy would also augment the effect of drugs in nonresponders to drug treatment, but the study by Kampman et al. ${ }^{11}$ suggests that these patients may also benefit from combination therapy. Fourth, patients who received combination therapy had considerably more therapeutic contact hours than patients who received drug treatment. We intentionally chose this design because it enhances the generalizability to clinical practice. Furthermore, a study that used relaxation to control for the amount of therapeutic contact has shown that the duration of therapeutic contact has no effect on OCD symptoms. ${ }^{23}$ Fifth, for practical reasons, psychometric assessments in the first and second phases of the treatment were performed by different investigators. Although precautions were taken to maximize the interrater reliability, it cannot be ruled out that the increase of symptoms seen in the drug treatment group was partly due to a difference in assessment rather than to a deterioration of symptoms. An argument in favor of this explanation would be that the increase was seen only between baseline and first assessment.

Another important issue, not tested in this study, is the durability of the improvement. Several studies have shown that patients receiving behavior therapy fare better after treatment discontinuation than those treated with medication. ${ }^{24,25}$ Patients who had received behavior therapy or a combination therapy had lower relapse rates and a longer time to relapse when drug treatment was discontinued.

In conclusion, this study has validated the clinical practice recommendation indicating that patients with moderate-to-severe OCD who have responded to drug treatment may benefit from the addition of behavior therapy. The timing of this augmentation with behavior therapy played a limited role, but the optimal effect was observed immediately after drug response was attained.

Drug names: clomipramine (Anafranil and others), fluoxetine (Prozac and others), paroxetine (Paxil, Pexeva, and others), sertraline (Zoloft), venlafaxine (Effexor).

\section{REFERENCES}

1. Weissman MM, Bland RC, Canino GJ, et al. The cross national epidemiology of obsessive compulsive disorder. J Clin Psychiatry 1994;55 (suppl 3):5-10 
2. Kolada JL, Bland RC, Newman SC. Epidemiology of psychiatric disorders in Edmonton: obsessive-compulsive disorder. Acta Psychiatr Scand Suppl 1994;376:24-35

3. Expert Consensus Guideline Series: Treatment of ObsessiveCompulsive Disorder. J Clin Psychiatry 1997;58(suppl 4):2-72

4. van Balkom AJ, van Oppen P, Vermeulen AWA, et al. A meta-analysis on the treatment of obsessive-compulsive disorder: a comparison of antidepressants, behavior, and cognitive therapy. Clin Psychol Rev 1994;14: 359-381

5. Marks IM, Stern RS, Mawson D, et al. Clomipramine and exposure for obsessive-compulsive rituals, 1. Br J Psychiatry 1980;136:1-25

6. Marks IM, Lelliott P, Basoglu M, et al. Clomipramine, self-exposure and therapist-aided exposure for obsessive-compulsive rituals. Br J Psychiatry $1988 ; 152: 522-534$

7. Cottraux J, Mollard E, Bouvard M, et al. A controlled study of fluvoxamine and exposure in obsessive-compulsive disorder. Int Clin Psychopharmacol 1990;5:17-30

8. Foa EB, Liebowitz MR, Kozak MJ, et al. Randomized, placebocontrolled trial of exposure and ritual prevention, clomipramine, and their combination in the treatment of obsessive-compulsive disorder. Am J Psychiatry 2005;162:151-161

9. Hohagen F, Winkelmann G, Rasche-Ruchle H, et al. Combination of behavior therapy with fluvoxamine in comparison with behaviour therapy and placebo: results of a multicenter study. Br J Psychiatry Suppl 1998;35:71-78

10. van Balkom AJ, de Haan E, van Oppen P, et al. Cognitive and behavioral therapies alone versus in combination with fluvoxamine in the treatment of obsessive compulsive disorder. J Nerv Ment Dis 1998; 186:492-499

11. Kampman M, Keijsers GP, Hoogduin CA, et al. Addition of cognitive-behavior therapy for obsessive-compulsive disorder patients non-responding to fluoxetine. Acta Psychiatr Scand 2002;106:314-319

12. Goodman WK, Price LH, Rasmussen SA, et al. The Yale-Brown Obsessive Compulsive Scale, 1: development, use and reliability. Arch Gen Psychiatry 1989;46:1006-1011

13. Goodman WK, Price LH. Assessment of severity and change in obsessive-compulsive disorder. Psychiatr Clin North Am 1992;15: 861-869

14. American Psychiatric Association. Diagnostic and Statistical Manual of Mental Disorders, Fourth Edition. Washington, DC: American Psychiatric Association; 1994

15. Denys D, van der Wee N, van Megen HJ, et al. A double blind comparison of venlafaxine and paroxetine in obsessive-compulsive disorder. J Clin Psychopharmacol 2003;23:568-575

16. Hamilton M. Development of a rating scale for primary depressive illness. Br J Soc Clin Psychol 1967;6:278-296

17. Hamilton M. Diagnosis and rating of anxiety. Br J Psychiatry 1959;3:67-69

18. Ballenger JC. Treatment of anxiety disorders to remission. J Clin Psychiatry 2001;62(suppl 12):5-9

19. Greist JH. An integrated approach to treatment of obsessive compulsive disorder. J Clin Psychiatry 1992;53(suppl 4):38-41

20. The Pediatric OCD Treatment Study (POTS) Team. Cognitive-behavior therapy, sertraline, and their combination for children and adolescents with obsessive-compulsive disorder. JAMA 2004;292:1969-1975

21. Nestadt G, Samuels J, Riddle MA, et al. The relationship between obsessive-compulsive disorder and anxiety and affective disorder: results from the Johns Hopkins OCD family study. Psychol Med 2001;31:481-487

22. Carter AS, Pollock RA, Suvak MK, et al. Anxiety and major depression comorbidity in a family study of obsessive-compulsive disorder. Depress Anxiety 2004;20:165-174

23. Lindsay M, Crino R, Andrews G. Controlled trial of exposure and response prevention in obsessive-compulsive disorder. Br J Psychiatry 1997; 171:135-139

24. Kordon A, Kahl KG, Broocks A. Clinical outcome in patients with obsessive-compulsive disorder after discontinuation of SRI treatment: results from a two-year follow-up. Eur Arch Psychiatry Clin Neurosci 2005;255:48-50

25. Simpson HB, Liebowitz MR, Foa EB, et al. Post-treatment effects of exposure therapy and clomipramine in obsessive-compulsive disorder. Depress Anxiety 2004;19:225-233 